C. УЛЬМ

\title{
О НЕКОТОРЫХ ПРИМЕНЕНИЯХ ПРИНЦИПА ПРОГНОЗИРОВАНИЯ ВЗАИМОДЕЙСТВИИ
}

Для решения задач нелинейного планирования устанавливается связь между принципом прогнозирования взакмодействий $\left[{ }^{1}\right]$ и методом декомпозиции, основанным нұ частичной линеаризации подсистем $\left[{ }^{2}\right]$. Этот принцип применяется для декомпозиции одной экономической модели, изученной Т. Кронше $\left[{ }^{3,4}\right]$. При этом координируемость рассмаприваемых задач доказывается на основании необходимых и достаточных условий минимума. По содержанию настоящая работа примыкает к статье $\left[{ }^{5}\right]$.

1. Представим задачу нелинейного планирования в виде

$$
f\left(x_{1}, \ldots, x_{n}\right) \rightarrow \min _{x \in X}
$$

где

$$
X=X_{1} \times \ldots \times X_{n},
$$

т. е. $x_{i} \in X_{i} ; x_{i}=\left(x_{i 1}, \ldots, x_{i n_{t}}\right)$ - в общем векторы.

Разложим задачу (1)-(2) некоторым образом на подзадачи

$$
f_{i}\left(x_{i}, \alpha_{i}\right) \rightarrow \min _{x_{t} \in X_{t}} \quad(i=1, \ldots, n) .
$$

При фиксированном $\alpha_{i}$ обозначим решение (не обязательно единственное) $i$-й подзадачи (3) через $x_{i}\left(\alpha_{i}\right)(i=1, \ldots, n)$.

Задача второго уровня (центра) состоит в нахождении координирующих входов $\hat{\alpha}_{i}$ таких, чтобы

$$
x_{i}\left(\hat{\alpha}_{i}\right)=\hat{x}_{i} \quad(i=1, \ldots, n),
$$

где $\hat{x}=\left(\hat{x}_{1}, \ldots, \hat{x}_{n}\right)$ - решение задачи $(1)-(2)$.

Для осуществления процесса координации введем (ср. $\left.\left[{ }^{1,5}\right]\right)$ т. н. функции 'взаимодействия $K_{i}(x)(i=1, \ldots, n)$, связывающие подзадачи (3) с общей задачей (1)-(2). Пусть $\left\{\alpha_{i}\right\}=K_{i}(X)(i=1, \ldots, n)$. Для каждого $\alpha$ можно вычислить значения $K_{i}(x(\alpha))$, где $x(\alpha)=$ $=\left(x_{1}\left(\alpha_{1}\right), \ldots, x_{n}\left(\alpha_{n}\right)\right)-$ некоторый вектор решения подзадач.

Будем говорить (ср. $\left.\left[{ }^{1,5}\right]\right)$, что при выбранных $f_{i}\left(x_{i}, \alpha_{i}\right)$ и $K_{i}(x)$

а) к задаче (1)-(2) применим принцип прогнозирования взаимодействий, если из предположения выполнения равенств

$$
\alpha_{i}=K_{i}(x(\alpha)) \quad(i=1, \ldots, n)
$$

следует

$$
x(\alpha)=\hat{x}
$$


б) задача (1)-(2) координируема с помощью принципа прогнозирования взаимодействий, если этот принцип применим и существуют $\alpha_{i}$ такие, при которых равенства (5) выполняются.

2. Пусть целевая функция (1) дана в виде

$$
f\left(x_{1}, \ldots, x_{n}\right)=\sum_{i=1}^{n} g_{i}\left(x_{i}\right)+g\left(x_{1}, \ldots, x_{n}\right) .
$$

Допустим, что функции $g(x)$ и $g_{i}\left(x_{i}\right)$ выпуклы и дифференцируемы, а множества $X_{i}-$ выпуклые компакты $(i=1, \ldots, n)$.

Выберем *

$$
f_{i}\left(x_{i}, \alpha_{i}\right)=g_{i}\left(x_{i}\right)+\left\langle\alpha_{i}, x_{i}\right\rangle
$$

и

$$
K_{i}(x)=\frac{\partial g\left(x_{1}, \ldots, x_{n}\right)}{\partial x_{i}} \quad(i=1, \ldots, n) .
$$

Тогда условия координации (5) примут вид

$$
\alpha_{i}=\frac{\partial g\left(x_{1}\left(\alpha_{1}\right), \ldots, x_{n}\left(\alpha_{n}\right)\right)}{\partial x_{i}} \quad(i=1, \ldots, n) .
$$

Покажем, что при выбранных $f_{i}\left(x_{i}, \alpha_{i}\right)$ и $K_{i}(x)$ задача (1)-(2) с целевой функцией (7) координируема с помощью принципа прогнозирования взаимодействий.

А. Применим ость принци па. Допустим, что условия координации (10) при некотором значении $\alpha$ выполнены. Тогда на основании необходимых и достаточных условий минимума (см., напр., [ $\left.{ }^{6}\right]$ ) для подзадач справедливо

$$
\left\langle\frac{\partial g_{i}\left(x_{i}\left(\alpha_{i}\right)\right)}{\partial x_{i}}+\frac{\partial g\left(x_{1}\left(\alpha_{1}\right), \ldots, x_{n}\left(\alpha_{n}\right)\right)}{\partial x_{i}}, x_{i}-x_{i}\left(\alpha_{i}\right)\right\rangle \geqslant 0
$$

для каждого $x_{i} \in X_{i}(i=1, \ldots, n)$. Суммируя эти неравенства, получим

$$
\langle\operatorname{grad} f(x(\alpha)), x-x(\alpha)\rangle \geqslant 0
$$

для каждого $x \in X$, т. е. необходимые и достаточные условия минимума для нашей задачи выполнены. Итак, из равенств (5) следует формула (6).

Б. Координируемость. Покажем, что система (10) имеет решение. Допустим, что решением ее является

$$
\alpha_{i}=\hat{\alpha}_{i}=\frac{\partial g\left(\hat{x}_{1}, \ldots, \hat{x}_{n}\right)}{\partial x_{i}} \quad(i=1, \ldots, n) .
$$

Это значит, что решением подзадачи

$$
f_{i}\left(x_{i}, \hat{\alpha}_{i}\right)=g_{i}\left(x_{i}\right)+\left\langle\frac{\partial g\left(\hat{x}_{1}, \ldots \hat{x}_{n}\right)}{\partial x_{i}}, x_{i}\right\rangle \rightarrow \min _{x_{i} \in x_{i}}
$$

\footnotetext{
* Символ $\langle\cdot, \cdot\rangle$ означает скалярное произведение,
} 
является

$$
x_{i}\left(\hat{\alpha}_{i}\right)=\hat{x}_{i} \quad(i=1, \ldots, n) .
$$

При нашем допущении и на основании необходимых и достаточных условий минимума должны быть справедливыми неравенства

$$
\begin{array}{r}
\left\langle\frac{\partial g_{i}\left(\hat{x}_{i}\right)}{\partial x_{i}}+\frac{\partial g\left(\hat{x}_{1}, \ldots, \hat{x}_{n}\right)}{\partial x_{i}}, x_{i}-\hat{x}_{i}\right\rangle \geqslant 0 \\
\left(x_{i} \in X_{i} ; i=1, \ldots, n\right) .
\end{array}
$$

Это действительно так, поскольку на основании условий минимума для расоматриваемой задачи

$$
\sum_{i=1}^{n}\left\langle\frac{\partial g_{i}\left(\hat{x}_{i}\right)}{\partial x_{i}}+\frac{\partial g\left(\hat{x}_{1}, \ldots, \hat{x}_{n}\right)}{\partial x_{i}}, x_{i}-\hat{x}_{i}\right\rangle \geqslant 0
$$

откуда следует выполняемость (16) для каждого $i=1, \ldots, n$. Допустим противное. Пусть по крайней мере для одного $i$, например, для $i=k$, существует точка $\tilde{x}_{k} \in X_{k}$ такая, что

$$
\left\langle\frac{\partial g\left(\hat{x}_{k}\right)}{\partial x_{k}}+\frac{\partial g\left(\hat{x}_{1}, \therefore, \hat{x}_{n}\right)}{\partial x_{k}}, \tilde{x}_{k}-\bar{x}_{k}\right\rangle<0 .
$$

Рассмотрим точку

$$
\tilde{x}=\left(\hat{x}_{1}, \ldots, \hat{x}_{k-1}, \tilde{x}_{k}, \hat{x}_{k+1}, \ldots, \hat{x}_{n}\right) \in X .
$$

Тогда, очевидно, $\left.\operatorname{grad} f\left(\hat{x}_{k}\right), \tilde{x}-\hat{x}\right\rangle<0$, т. е. $\tilde{x}$ не удовлетворяет условиям (17). Мы получили противоречие. Итак, система (10) имеет решение и наша задача является координируемой.

3 а меч а н ие. Аналогичный метод декомпозиции (без ограничения $x \in X)$, но с другой точки зрения, был представлен в $\left[{ }^{2}\right]$. Там же был разработан и некоторый алгоритм для координации, т. е. для решения системы (10). Вышеизложенное показывает, что этот метод декомпозиции можно интерпретировать как пример применения принципа прогнозирования взаимодействий.

3. Рассмотрим применение этого принципа для декомпозиции одной экономической модели, исследованной другими способами Т. Кронше $\left[{ }^{3,4}\right] * *:$

$$
\begin{aligned}
& \sum_{i=0}^{n} f_{i}\left(x_{i}\right) \rightarrow \min , \\
& g_{i}\left(x_{0}\right)+h_{i}\left(x_{i}\right) \leqslant b_{i} \quad(i=1, \ldots, n), \\
& x_{i} \in X_{i} \quad(i=0, \ldots, n) .
\end{aligned}
$$

Выберем

$$
K_{i}(x)=h_{i}\left(x_{i}\right) \quad(i=1, \ldots, n)
$$

и построим подзадачи следующим образом.

** В $\left[{ }^{3,4}\right]$ можно найти и экономическую интерпретащию модели (19). 
1-я подзадача:

$$
\begin{aligned}
& f_{0}\left(x_{0}\right) \rightarrow \min , \\
& g_{i}\left(x_{0}\right)+\alpha_{i} \leqslant b_{i} \quad(i=1, \ldots, n), \\
& x_{0} \in X_{0} ;
\end{aligned}
$$

$i$-я подзадача $(i=2, \ldots, n+1)$ :

$$
\begin{aligned}
& f_{i-1}\left(x_{i-1}\right)+\beta_{i-1} h_{i-1}\left(x_{i-1}\right) \rightarrow \min , \\
& x_{i-1} \in X_{i-1} .
\end{aligned}
$$

Координирующими входами второго уровня являются $\alpha=\left(\alpha_{1}, \ldots, \alpha_{n}\right)$ и $\beta=\left(\beta_{1}, \ldots, \beta_{n}\right)$ (при этом с помощью величин $\beta_{i} \geqslant 0$ модифицируются целевые функции подсистем). Предположим, что $f_{i}, g_{i}, h_{i}$ выпуклые функции, $X_{i}$ - выпуклые компакты, а $x_{i}-$ в общем векторы. Допустим также, что для глобальной задачи (19) и для 1-й подзадачи (при интересующих нас значениях $\alpha_{i}$ ) условие Слейтера выполняется. Обозначим решение 1-й подзадачи через $x_{0}(\alpha)$, соответствующие множители Лагранжа через $\lambda_{i}(\alpha)(i=1, \ldots, n)$ и решение $i$-й подзадачи џерез $x_{i-1}\left(\beta_{i-1}\right) \quad(i=2, \ldots, n+1)$. Оказывается, что условия жоординации выражаются в виде

$$
\begin{aligned}
\alpha_{i} & =h_{i}\left(x_{i}\left(\beta_{i}\right)\right), \\
\beta_{i} & =\lambda_{i}(\alpha) \\
(i & =1, \ldots, n) .
\end{aligned}
$$

4. Покажем, что при сделанных предположениях задача (19) координируема с помощьо принципа прогнозирования взаимодействий.

А. Применим ость принци па. При наших предположениях задача (19) и подзадачи (20), (21) имеют решение, причем для задач (19) и (20) необходимым и. достаточным условием оптимальности является существование седловой точки у соответствующих функций Лагранжа. Допустим, что существуют $\bar{\alpha}$ и $\bar{\beta}$ такие, что условия (22) выполняются. Покажем, "то тогда $\bar{x}=\left(x_{0}(\bar{\alpha}), \quad x_{1}\left(\overline{\beta_{1}}\right), \ldots, x_{n}\left(\bar{\beta}_{n}\right)\right)=$ $=\left(\bar{x}_{0}, \ldots, \bar{x}_{n}\right)$ и $\bar{\lambda}=\left(\lambda_{1}(\bar{\alpha}), \ldots, \lambda_{n}(\bar{\alpha})\right)=\left(\bar{\lambda}_{1}, \ldots, \bar{\lambda}_{n}\right)$ являются соответственно решением задачи (19) и вектором множителей Лагранжа.

Условия седловой точки для 1-й подзадачи при $\bar{\alpha}_{i}=h_{i}\left(\bar{x}_{i}\right)$ и $\bar{\beta}_{i}=\bar{\lambda}_{i}$ выражаются в виде

$$
\begin{aligned}
& f_{0}\left(\bar{x}_{0}\right)+\sum_{i=1}^{n} \lambda_{i}\left(g_{i}\left(\bar{x}_{0}\right)+h_{i}\left(\bar{x}_{i}\right)-b_{i}\right) \leqslant \\
& \leqslant f_{0}\left(\bar{x}_{0}\right)+\sum_{i=1}^{n} \bar{\lambda}_{i}\left(g_{i}\left(\bar{x}_{0}\right)+h_{i}\left(\bar{x}_{i}\right)-b_{i}\right) \leqslant \\
& \leqslant f_{0}\left(x_{0}\right)+\sum_{i=1}^{n} \bar{\lambda}_{i}\left(g_{i}\left(x_{0}\right)+h_{i}\left(\bar{x}_{i}\right)-b_{i}\right) \\
&\left(x_{0} \in X_{0} ; \lambda_{i} \geqslant 0, i=1, \ldots, n\right) .
\end{aligned}
$$

Условия оптимальности для $i$-й подзадачи при $\boldsymbol{\beta}_{i-1}=\bar{\lambda}_{i-1}$ $(i=2, \ldots, n+1)$ :

$$
\begin{gathered}
f_{i-1}\left(\bar{x}_{i-1}\right)+\bar{\lambda}_{i-1} h_{i-1}\left(\bar{x}_{i-1}\right) \leqslant f_{i-1}\left(x_{i-1}\right)+\bar{\lambda}_{i-1} h_{i-1}\left(x_{i-1}\right) \\
\left(x_{i-1} € X_{i-1}\right),
\end{gathered}
$$


Из этих неравенств с помощью суммирования получим:

$$
\begin{aligned}
& \sum_{i=0}^{n} f_{i}\left(\bar{x}_{i}\right)+\sum_{i=1}^{n} \lambda_{i}\left(g_{i}\left(\bar{x}_{0}\right)+h_{i}\left(\bar{x}_{i}\right)-b_{i}\right) \leqslant \\
& \leqslant \sum_{i=0}^{n} f_{i}\left(\bar{x}_{i}\right)+\sum_{i=1}^{n} \bar{\lambda}_{i}\left(g_{i}\left(\bar{x}_{0}\right)+h_{i}\left(\bar{x}_{i}\right)-b_{i}\right) \leqslant \\
& \leqslant \sum_{i=0}^{n} f_{i}\left(x_{i}\right)+\sum_{i=1}^{n} \bar{\lambda}_{i}\left(g_{i}\left(x_{0}\right)+h_{i}\left(x_{i}\right)-b_{i}\right) \\
&\left(x_{i} \in X_{i}, i=0, \ldots, n ; \lambda_{i} \geqslant 0, i=1, \ldots, n\right) .
\end{aligned}
$$

Условия (25) являются условиями седловой точки для задачи (19). Следсвательно, $\bar{x}$ есть решение задачи (19), а $\bar{\lambda}-$ соответствующий вектор множителей Лагранжа. Итак, принцип прогнозирования взаимодействий применим.

Б. Координи р уем ость. Покажем, что система (22) имеет решение. Пусть $\hat{x}$ - решение задащи (19), а $\hat{\lambda}-$ соответствующий вектор множителей Лагранжа. Тогда при $\bar{x}=\hat{x}, \bar{\lambda}=\hat{\lambda}$ неравенства (25) выполняются. Выберем

$$
\begin{aligned}
& \alpha_{i}=h_{i}\left(\hat{x}_{i}\right), \\
& \beta_{i}=\hat{\lambda}_{i} \\
& (i=1, \ldots, n) .
\end{aligned}
$$

Тогда подзадачи примут следующий вид.

1-я подзадача:

$$
\begin{aligned}
& f_{0}\left(x_{0}\right) \rightarrow \min , \\
& g_{i}\left(x_{0}\right)+h_{i}\left(\hat{x}_{i}\right) \leqslant b_{i} \quad(i=1, \ldots, n) \\
& x_{0} \in X_{0},
\end{aligned}
$$

$i$-я подзадача $(i=2, \ldots, n+1)$ :

$$
\begin{aligned}
& f_{i-1}\left(x_{i-1}\right)+\hat{\lambda}_{i-1} h_{i-1}\left(x_{i-1}\right) \rightarrow \min \\
& x_{i-1} \in X_{i-1}
\end{aligned}
$$

Мы должны показать, что решением 1 -й подзадачи является $\hat{x}_{0}$, соответствующим вектором множителей. Лагранжа $-\hat{\lambda}$ и решением $i$-й подзадачи - $\hat{x}_{i-1}(i=2, \cdots, n+1)$. Тогда для 1 -й подзадачи должны иметь место следующие условия седловой точки:

$$
\begin{aligned}
& f_{0}\left(\hat{x}_{0}\right)+\sum_{i=1}^{n} \lambda_{i}\left(g_{i}\left(\hat{x}_{0}\right)+h_{i}\left(\hat{x}_{i}\right)-b_{i}\right) \leqslant \\
& \leqslant f_{0}\left(\hat{x}_{0}\right)+\sum_{i=1}^{n} \hat{\lambda}_{i}\left(g_{i}\left(\hat{x}_{0}\right)+h_{i}\left(\hat{x}_{i}\right)-b_{i}\right) \leqslant \\
& \leqslant f_{0}\left(x_{0}\right)+\sum_{i=1}^{n} \hat{\lambda}_{i}\left(g_{i}\left(x_{0}\right)+h_{i}\left(x_{i}\right)-b_{i}\right) \\
& \left(x_{0} \in X_{0} ; \lambda_{i} \geqslant 0, i=1, \ldots, n\right) .
\end{aligned}
$$


Первое из неравенств (29) выполняется, поскольку $\hat{\lambda}_{i}\left(g_{i}\left(\hat{x}_{0}\right)+\right.$ $\left.+h_{i}\left(\hat{x_{i}}\right)-b_{i}\right)=0$ и $\lambda_{i}\left(g_{i}\left(\hat{x_{0}}\right)+h_{i}\left(\hat{x_{i}}\right)-b_{i}\right) \leqslant 0 \quad\left(\lambda_{i} \geqslant 0\right)$. Рассмотрим второе неравенство, которое можно представить в виде

$$
\begin{aligned}
f_{0}\left(\hat{x}_{0}\right)+\sum_{i=1}^{n} \hat{\lambda}_{i} g_{i}\left(\hat{x}_{0}\right) & \leqslant f_{0}\left(x_{0}\right)+\sum_{i=1}^{n} \hat{\lambda}_{i} g_{i}\left(x_{0}\right) \\
\left(x_{0}\right. & \left.\in X_{0}\right) .
\end{aligned}
$$

Вместе с неравенством (30) рассмотрим неравенства, которые должны иметь место для остальных подсистем:

$$
\begin{gathered}
f_{i}\left(\hat{x}_{i}\right)+\hat{\lambda}_{i} h_{i}\left(\hat{x}_{i}\right) \leqslant f_{i}\left(x_{i}\right)+\hat{\lambda}_{i} h_{i}\left(x_{i}\right) \\
(i=1, \ldots, n) .
\end{gathered}
$$

Выполняемость неравенств (30) и (31) следует из условий (25), где $\bar{x}=\hat{x}$ и $\bar{\lambda}=\hat{\lambda}$. На основании последних

$$
\begin{array}{r}
\sum_{i=0}^{n} f_{i}\left(\hat{x}_{i}\right)+\sum_{i=1}^{n} \hat{\lambda}_{i} g_{i}\left(\hat{x}_{0}\right)+\sum_{i=1}^{n} \hat{\lambda}_{i} h_{i}\left(\hat{x}_{i}\right) \leqslant \\
\leqslant \sum_{i=0}^{n} f_{i}\left(x_{i}\right)+\sum_{i=1}^{n} \hat{\lambda}_{i} g_{i}\left(x_{0}\right)+\sum_{i=1}^{n} \hat{\lambda}_{i} h_{i}\left(x_{i}\right) \\
\left(x_{i} \in X_{i} ; \quad i=0, \ldots, n\right) .
\end{array}
$$

Действительно, допустим, что существует точка $\tilde{x}_{0} \in X_{0}$ такая, что

$$
f_{0}\left(\tilde{x}_{0}\right)+\sum_{i=1}^{n} \hat{\lambda}_{i} g_{i}\left(\tilde{x}_{0}\right)<f_{0}\left(\hat{x}_{0}\right)+\sum_{i=1}^{n} \hat{\lambda}_{i} g_{i}\left(\hat{x}_{0}\right)
$$

Тогда точка $\left(\tilde{x}_{0}, \hat{x}_{1}, \ldots, \hat{x}_{n}\right) \in X=X_{0} \times \ldots \times X_{n}$ не удовлетворяет условиям (32), т. е. мы получили противоречие. Если положить, что при $1 \leqslant i=k \leqslant n$ существует точка $\tilde{x}_{k} \in X_{k}$ такая, что

$$
f_{k}\left(\tilde{x}_{k}\right)+\hat{\lambda}_{k} h_{k}\left(\tilde{x}_{k}\right)<f_{k}\left(\hat{x}_{k}\right)+\hat{\lambda}_{k} h_{k}\left(\hat{x}_{k}\right),
$$

то точка $\left(\hat{x}_{0}, \ldots, \hat{x}_{k-1}, \tilde{x}_{k}, \hat{x}_{k+1}, \ldots, \hat{x}_{n}\right) \in X$ не удовлетворяет условиям (32), т. е. мы опять получили противоречие. Следовательно, выполняются и неравенства (30), (31). Итак, задача (19) является координируемой.

5. Аналогичным образом можно доказать координируемость задачи (19) с помощью принципа прогнозирования взаимодействий, если выбрать

$$
K_{i}(x)=g_{i}\left(x_{0}\right) \quad(i=1, \ldots, n)
$$

и построить подзадачи следующим образом.

1-я подзадача:

$$
\begin{aligned}
& f_{0}\left(x_{0}\right)+\sum_{i=1}^{n} \beta_{i} g_{i}\left(x_{0}\right) \rightarrow \min , \\
& x_{0} \in X_{0}
\end{aligned}
$$

(решение 1-й подзадачи обозначим через $x_{0}(\beta)$ ); 
$i$-я подзадача $(i=2, \ldots, n+1)$ :

$$
\begin{aligned}
& f_{i-1}\left(x_{i-1}\right) \rightarrow \min , \\
& h_{i-1}\left(x_{i-1}\right)+\alpha_{i-1} \leqslant b_{i-1}, \\
& x_{i-1} \in X_{i-1}
\end{aligned}
$$

(решение $i$-й подзадачи обозначим через $x_{i-1}\left(\alpha_{i-1}\right)$, соответствующий множитель Лагранжа $\left.\lambda_{i-1}\left(\alpha_{i-1}\right)\right)$. Условия координации выражаются в данном случае в виде

$$
\begin{aligned}
& \alpha_{i}=g_{i}\left(x_{0}(\beta)\right), \\
& \beta_{i}=\lambda_{i}\left(\alpha_{i}\right) \\
& (i=1, \ldots, n) .
\end{aligned}
$$

6. При декомпозиции модели (19) с помощью принципа прогнозирования взаимодействий мы имеем дело с т. н. бикоординацией, т. е. второй уровень (центр) модифицирует в ходе вычислений как целевые функции, так и ограничения соответствующих подсистем до тех пор, пока условия координации ((22) или (38)) не будут выполненными. В данной статье вопрос о способах координации не обсуждается. Отметим толыко, что разработанные здесь схемы декомпозиции можно перенести и на те случаи, когда вместе с оптимальным решением задачи (19) требуется найти и соответствующие множители Лагранжа.

Рассмотрим простой пример, заимствованный из [3]:

$$
\begin{aligned}
& 4\left(x_{0}-10\right)^{2}+\left(x_{1}-10\right)^{2} \rightarrow \min , \\
& \left(x_{0}-6\right)^{2}-4\left(x_{1}-5\right)+20 \leqslant 0, \\
& 0 \leqslant x_{0}, x_{1} \leqslant 20 .
\end{aligned}
$$

В силу выражений (20) и (21) подзадачи принимают вид

$$
\begin{array}{ll}
4\left(x_{0}-10\right)^{2} \rightarrow \min & \left(x_{1}-10\right)^{2}-4 \beta\left(x_{1}-5\right) \rightarrow \min \\
\left(x_{0}-6\right)^{2}+\alpha \leqslant-20 & 0 \leqslant x_{1} \leqslant 20, \\
0 \leqslant x_{0} \leqslant 20 &
\end{array}
$$

причем $K_{1}(x)=-4\left(x_{1}-5\right)$.

В данном случае подзадачи можно решить аналитически, а именно:

$$
\begin{gathered}
x_{0}(\alpha)= \begin{cases}10, & \text { если } \alpha<-36, \\
6+\sqrt{-(\alpha+20)}, & \text { если }-36 \leqslant \alpha \leqslant-20, \\
\text { не существует, если } & \alpha>-20 ;\end{cases} \\
\lambda_{1}(\alpha)= \begin{cases}0, & \text { если } \alpha<-36, \\
\sqrt{\frac{256}{-(\alpha+20)}-4,} & \text { если }-36 \leqslant \alpha<-20, \\
\text { не существует, если } \alpha \geqslant-20 ;\end{cases} \\
x_{1}(\beta)= \begin{cases}10+2 \beta, & \text { если } 0 \leqslant \beta \leqslant 5, \\
20, & \text { если } \beta \geqslant 5 .\end{cases}
\end{gathered}
$$

По (22) условие координации выражается в виде:

$$
f(\alpha) \equiv \alpha+4 x_{1}\left(\lambda_{1}(\alpha)\right)-20=0 .
$$


Для решения этого уравнения был применен метод хорд, причем в качестве начальных приближений были выбраны $\alpha_{0}=-60, \alpha_{1}=-21$. После восьми итераций были получены следующие результаты:

$$
\begin{aligned}
\alpha & =-29,5090027, \\
\lambda_{1}(\alpha) & =1,1886263 \approx \hat{\lambda}_{1}, \\
x_{0}(\alpha) & =9,0836671 \approx \hat{x}_{0}, \\
x_{1}(\beta)=x_{1}\left(\lambda_{1}(\alpha)\right) & =12,3772526 \approx \hat{x}_{1}, \\
f(\alpha) & =0,0000000 .
\end{aligned}
$$

\section{ЛИТЕРА Т У Р А}

1. Месарович М., Мако Д., Т акаха ра И., Теория иерархических многоуровневых систем, М., 1973.

2. Wierzbicki, A. P., Preprints of IFAC 5th World Congress, Paris, June 12-17, $1972,3 \mathrm{~B}, 30.5$.

3. Krons jö T., Economics of Planning, 9, No. 1-2, 71 (1969).

4. К р онше Т., В кн.: Оптимизация, вып. 12 (29), Новосибирск, 1973, с. 127.

5. У л ь м С., Изв. АН ЭССР, Физ. Матем., 23, 319 (1974).

6. Л евитин Е. С., Поляк Б. Т., Ж. вычисл. матем. и матем. фмзики, 6, 787 (1966).
Ннститут кибернетики
Академии наук Эстонской ССР
Поступила в редакцию
4/XI 1976

\section{S. $U L M$}

\section{MONINGATEST KOOSTOOO ENNUSTAMISE PRINTSIIBI RAKENDUSTEST}

Artiklis esitatakse seos koostöö ennustamise printsiibi $\left[{ }^{1}\right]$ ja A. P. Wierzbicki dekompositsioonimeetodi $\left.{ }^{[2}\right]$ vahel. Mainitud printsiipi on rakendatud ka T. Kronsjö käsitletud majandusmudeli $\left[{ }^{3,4}\right]$ dekompositsiooniks. Seejuures on tōestatud ülesannete koordineeruvus miinimumi tarvilike ja piisavate tingimuste abil.

\section{S. $U L M$}

\section{ON SOME APPLICAT!ONS OF THE INTERACTION PREDICTION PRINCIPLE}

A conneclion between the interaction prediction principle $\left[{ }^{1}\right]$ and the decomposition method proposed by A. P. Wierzbicki $\left[{ }^{2}\right]$, is established. This principle is also applied for decomposition of an economic model investigated by $\mathrm{T}$. Kronsjö $\left[{ }^{3,4}\right]$. The coordinability is proved via the necessary and sufficient conditions for minima. 